\title{
Sudden Infant Death Syndrome: A Case Report in Pakistan
}

\author{
Mishal Liaqat* \\ MS. Nursing Student, University of Lahore, Pakistan \\ Sana Sehar \\ Assistant Professor, University of Lahore, Pakistan \\ Muhammad Afzal \\ Assistant Professor, University of Lahore, Pakistan
}

\begin{abstract}
Sudden infant death syndrome (SIDS) is the sudden death of an infant without any identified cause after a complete autopsy report, death scene investigation and from parent's history. There is little published literature or statistics present in a developing country like Pakistan. So the aim of this study was to identify those factors that are associated with Sudden infant death syndrome in relation to Pakistani culture. The case report was based on a case of two months old baby previously healthy was found dead on his crib. The autopsy report and investigation of the scene giving non-specified symptoms further, diagnose the case as Sudden infant death syndrome. The study found that in our culture early marriages, inadequate knowledge regarding child-rearing and traditional use of Quilts for children are the major contributing factors in the prevention of Sudden infant death syndrome.
\end{abstract}

Keywords: Infant Death; Sudden; Crib Death.

DOI: $10.7176 / \mathrm{JMPB} / 62-04$

Publication date: December $31^{\text {st }} 2019$

\section{Introduction}

Sudden Infant death syndrome (SIDS) was first reported in 1953 and is defined as the sudden death of an infant (under one year) that remained unexplained after a complete autopsy report and investigation of the death scene (1). SIDS is also called "CRIB DEATH" as most of the deaths were occurring in the crib during the sleeping hours of the infant. Since it is called a syndrome means, that it is under the influence of a number of disorders including respiratory, cardiovascular and abnormalities in part of the brain that control breathing and arousal events in the infant. It is the leading cause of death in infants under one year of age and most events occur in 1-6 months of age.

All over the world, it is a mystery because of unknown cause and mostly relies on medical hypothesis (2). SIDS rate in 2005 in Japan is 0.16 per 1000, in the United State; it is 0.54 per 1000 live births (3). New Zealand reported as highest incidence rate of SIDS as 0.80 per 1000 live births (4). Shockingly, the rate of SIDS in developing countries like Pakistan is unknown. The factors associated with SIDS are modified and nonmodifiable. The non-modifiable risk factors including male sex, Sleep apnea, Cardiovascular, respiratory, and any abnormality in brain stem (5). Although there are a number of modifiable risk factors associated with SIDS including prone positioning, Parental smoking, alcohol, overheating, prematurity, underweight, and adult bedsharing. Identification and decreasing these factors leading to a decrease in SIDS cases in the last two decades (6). A "Back to Sleep" campaign in 1990 leading to a number of decreasing cases (7). But still, the ratio is high in developing countries like Pakistan in which people are less aware of this condition.

According to the best of author knowledge, there is little published literature and few cases reported on SIDS in Pakistan. There is no such protocol of educating parents after the birth of children regarding the factors leading to SIDS. The presentation purpose of this case report is to emphasize the importance of this hidden disorder that is silently killing many infants all over the world. The main aim of this report was to identify what are the major risks factors related to our culture that is contributing to SIDS?

\section{Case Report}

A case was reported in January 2018 in the pediatric emergency of Jinnah hospital, Lahore. In which a 2 months old baby previously reported as healthy was found to be dead on his crib. After examination, the baby was found incomplete cardiac arrest and was found dead on his arrival in the emergency. Both parents were present at that time and are in a state of shock. According to the mother-baby was healthy with a mild complaint of flu and cough at night. She gives him cow milk in the bottle at 11:30 pm at night and wrapped in Quilt before sleeping. The quilt was placed in such manner that half was under the baby and a half was over him to keep him warm. She did not feel anything special and serious regarding child health. In the morning at 6:00 pm, she found that the infant was not responding and found something wrong. According to mother, she found him in a side-lying 
position and quilt was covered over his head. His hands clenched tightly to the quilt. His pamper was full of stool. There was no sign of vomiting in the crib. She was sleeping near the child and don't feel or listen to any crying at night. The infant was born at term through spontaneous vaginal delivery in the Jinnah hospital labor room. Mother age is 15 years and the father was 19 years old. Soon after two months of her marriage, she got conceived. She did not suffer any problem during pregnancy and antenatal visits are followed. After the delivery baby was healthy and having a weight of $2.8 \mathrm{~kg}$ and a length of 48 centimeters. She followed the routine vaccination schedule of the baby and reported mild fever after vaccination for a few days. Baby was not recently received any vaccination before death. Mother was a housewife and the child's father was working in a store having lower socio-economic status. The case was reported to the local police and on the investigation; they concluded that they live in a small congested house. The infant sleeping environment consists of a crib which is consists of a small soft pillow and two quilts of soft stuff that covered the whole crib. There is a small window in the room which was found close due to winter season. The forensic autopsy report revealed that the infant had edema in the lungs and brain. The internal organs also showed signs of edema and cyanosis. There was no obstruction found in larynx and not find any sign of aspiration. There was no sign of physical abuse and violence child body. All these findings are non-specific and leading to a natural death due to SIDS, as supported by literature found. The infant was 53 centimeters in length and $3.5 \mathrm{~kg}$ weight on death having. Parents reported no family history of such kind of incidents in the past.

\section{Discussion}

Deaths in infants due to SIDS is alarming and challenging for health professionals, parents, and community due to sudden unidentified cause in previously otherwise healthy baby. Although the number of SIDS in a developed country has been significantly reduced due to back to sleep campaign and education of people regarding risk factors. But still is challenging in developing countries like Pakistan in which inadequate reporting or sufficient data of infant died trough SIDS is lacking (8).

In the above case, there are a number of risk factors that were found associated with SIDS. First, both parents are age below twenty which showed their lack of competency and skill towards childcare. A case-control study in Brazil was conducted to identify risk factors related to SIDS in a developing country and concluded that there is a link between maternal age and incidence of SIDS especially if age is below twenty (9). The second baby was male and the family's socioeconomic status was poor as evidenced by a small house with no adequate ventilation in the room. This also associated with the SIDS in literature (10). Thirdly mother notices that the child complains of flu and cough. AS infants are nose breather so there may be the possibility of nose congestion and difficulty in breathing at night. And last but not least the most important factor was the use of a quilt that was not meeting the standard of child protection. As in the case, Quilt was found soft and covered the child from under and above over the head. So it may be the possibility that the child was sucked in the quilt unable to breathe and suffocated as autopsy report revealed edema in lungs. Literature showed that there is a risk of SIDS using soft surface mattress (11).

This case also matches the criteria of the triplet model that describe there is the first vulnerability of infant due to some disease condition and prematurity, here flu is the vulnerability factor. Second infant critical period of development related to first months of growth and incompetency and thirdly some external factors like prone position, bedding or soft quilt leading to SIDS. Here in our case use of Quilt was the third leading factor (12). In Pakistani culture, parents are responsible for their children. There is no fashion to place small children in separate rooms as compared to other developed countries. Most people are abstaining from alcohol due to religious constraints. There is no tradition of women smoking in our culture. So a number of risk factors are minimized in our culture (13). But still, there are other factors that should be focused such as poverty, lack of knowledge regarding childcare, early marriages especially in girls, and most importantly using soft quilts. There is a tradition of gifting newborn sleeping set consists of pillow, mattress, and quilt by some close relatives. Often that sleeping set did not match the standard for the child especially when the child is under one-year-old and incompetent to remove quilt in case of accidentally covering his face at night. As research showed that the head is an important source of heat removal and the number of deaths in SIDS is due to the covering of head (14).

American Academy of Pediatrics recommends that all infants should be provided a safe sleep environment including back to sleep position, removing all toys, room sharing, adequate temperature and avoidance of soft surface or overheating (15). So it is a dire need for a collaborative program in which health departments, parents, the community should be involved to educate people regarding these factors and their prevention. In addition, there should be proper recording and reporting of such cases is important. Analyzing or interpretation will lead further to identify and compare each case to rule out factors related to our culture that expose children at risk of SIDS. There should be a discharge written plan from the labour room in every government and private hospitals that educate parents about the prevention of such factors. Such education is especially important in vulnerable newborns (born with some health problems, premature and underweight) and vulnerable families (parents age, socioeconomic status, and education). Health ministry should take action to have control over marketing a 
standard sleeping set for infants that meet the international standards for child safety and protection (16).

\section{Conclusion}

In conclusion, there are a number of factors leading to SIDS all over the world. These factors are under the influence of cultural variations and practices that are important to identify. In developing countries like Pakistan early marriages, poverty, inadequate knowledge about safety measures of infant and traditional use of sleeping sets that are not meeting the international standards are the leading cause of SIDS. Moreover, parent's education regarding these factors is important to decrease such incidents.

\section{Conflict Of Interest}

There was no conflict of interest among authors.

\section{Ethical Approval}

The study was approved by the ethical committee of Allama Iqbal medical college, Jinnah hospital Ethical Review board (EX- 23-7-92).

\section{Patient Consent}

Informed consent was taken from child parents of the study

\section{Authors Contribution}

LM Conceived the idea and did bench work in writing the manuscript, SS helped in designing the case report and facilitated, LI supervised the case report and finalize the manuscript. ALR helped in compiling data and help in reporting.

\section{Acknowledgement}

We specifically thank the School of nursing, the University of Lahore for helping us in this study.

\section{References}

1. Mitchell, E. A., \& Krous, H.F. (2015). Sudden unexpected death in infancy: a historical perspective. Journal of Paediatric and Child Health, 51, 108-112.

2. Elhaik, E. (2016). A "Wear and Tear" Hypothesis to Explain Sudden Infant Death Syndrome: Hypothesis and Theory Article. Available https://doi.org/10.3389/fneur.2016.00180 (October 28, 2016).

3. Hauck, F. R., \& Tanabe, K.O. (2008). International trends in sudden infant death syndrome: stabilization of rates requires further action. Pediatrics, 122, 660-666.

4. Moon, R. Y., Horne, R. S., \& Hauck, F. R. (2007). Sudden infant death syndrome. Lancet, 370, 1578- 1587

5. Garstang, J., Ellis, C., \& Sidebotham. P. (2015). An evidence-based guide to the investigation of sudden unexpected death in infancy. Forensic Science, Medicine, and Pathology, 11, 345-357.

6. Goldstein, R. D., Trachtenberg, F. L., Sens, M. A., Harty, B. J., \& Kinney, H. C. (2016). Overall postneonatal mortality and rates of SIDS. Pediatrics, 137, 1-10.

7. Waters, K. A., (2014). SIDS symposium-a perspective for future research. Paediatr Respiratory Reviews, $15,285-286$.

8. Shaikh, F. H., Usman, M. S., \& Salman, S. A. (2017). Sudden infant death syndrome - A medical mystery. Journal of Pakistan Medical Association, 67(10).

9. Pinho, A. P., Aerts, D., \& Nunes, M. L. (2008). Risk factors for sudden infant death syndrome in a developing country. Revista de Saúde Pública, 42(3).

10. Eschbach, K., \& Malloy, M. H. (2007). Association of poverty with sudden infant death syndrome in metropolitan counties of the United States in the years 1990 and 2000. South Medicine Journal, 100(11), 1107-1113.

11. Kroll, M. E., Quigley, M. A., Kurinczuk, J. J., Dattani, N., Li, Y., \& Hollowell, J. (2018). Ethnic variation in unexplained deaths in infancy, including sudden infant death syndrome (SIDS), England and Wales 20062012: national birth cohort study using routine data. Journal of epidemiology and community health, 72(10), 911-918.

12. Filiano, J. J., \& Kinney, H. C., (1994). A perspective on neuropathologic findings in victims of the sudden infant death syndrome: the triple-risk model. Biology of the Neonate, 65(3-4), 194-7.

13. Crane, D., \& Ball, H. L., (2016). A qualitative study in parental perceptions and understanding of SIDSreduction guidance in a UK bi-cultural urban community. BMC Pediatrics, 16.

14. Mitchell, A. E., Thompson, J. M., Becroft, D. M., Bajanowski, B., Brinkmann, B., Happe, A., Vennemann M. M. (2008). Head covering and the risk for SIDS: Findings from the New Zealand and German SIDS Case-control studies. Pediatrics, 121(6), 1478-1483. 
15. American Academy of Pediatrics. (2016). Task Force on Sudden Infant Death Syndrome.

16. SIDS and other sleep-related infant deaths: updated 2016 recommendations for a safe infant sleeping environment. Pediatrics, 138(5). 